\title{
Widening the landscape of heritable pulmonary hypertension mutations in paediatric and adult cases
}

\author{
Mélanie Eyries ${ }^{1,2}$, David Montani $\mathbb{1 0}^{3}$, Sophie Nadaud ${ }^{2}$, Barbara Girerd ${ }^{3}$, \\ Marilyne Levy ${ }^{4}$, Arnaud Bourdin ${ }^{5,6}$, Romain Trésorier ${ }^{7}$, Ari Chaouat $\mathbb{0}^{8}$, \\ Vincent Cottin ${ }^{(1)}{ }^{9}$, Céline Sanfiorenzo ${ }^{10}$, Grégoire Prevot ${ }^{11}$, \\ Martine Reynaud-Gaubert ${ }^{12}$, Claire Dromer ${ }^{13}$, Ali Houeijeh ${ }^{14}$, Karine Nguyen ${ }^{15}$, \\ Florence Coulet ${ }^{1}$, Damien Bonnet ${ }^{4}$, Marc Humbert $\mathbb{1}^{3}$ and Florent Soubrier (10 ${ }^{1,2}$
}

@ERSpublications

Gene panel sequencing unravels the genetic architecture of pulmonary hypertension in adult and paediatric cases, emphasises the importance of BMPR2, EIF2AK4, BMP9 and TBX4 mutations, and suggests BMP10 as a new gene for the disease http://ow.ly/Oxes30mXnrI

Cite this article as: Eyries M, Montani D, Nadaud S, et al. Widening the landscape of heritable pulmonary hypertension mutations in paediatric and adult cases. Eur Respir J 2019; 53: 1801371 [https://doi.org/ 10.1183/13993003.01371-2018].

\section{ABSTRACT}

Background: Heritable forms of pulmonary arterial hypertension (PAH) and pulmonary veno-occlusive disease/pulmonary capillary haemangiomatosis (PVOD/PCH) diverge by lung histopathological lesions, clinical and para-clinical presentation, their responsible genes, and mode of transmission. Since the identification of the BMPR2 gene in families affected by PAH, mutations in several other genes have been discovered for both forms. The mutation landscape in these new genes is not yet well known.

Methods: We set up a next-generation sequencing-based targeted sequencing gene panel allowing known genes for $\mathrm{PAH}$ and $\mathrm{PVOD} / \mathrm{PCH}$ to be analysed simultaneously. Genetic analysis was prospectively performed on $263 \mathrm{PAH}$ and $\mathrm{PVOD} / \mathrm{PCH}$ patients (adult and paediatric cases).

Results: Pathogenic mutations were identified in $19.5 \%$ of sporadic PAH patients $(n=180), 54.5 \%$ of familial PAH patients and $13.3 \%$ of $\mathrm{PVOD} / \mathrm{PCH}$ patients. BMPR2 was the most frequently mutated gene, followed by TBX4 in both paediatric and adult PAH. BMP9 mutations were identified in $1.2 \%$ of adult PAH cases. EIF2AK4 biallelic mutations were restricted to PVOD/PCH. A truncating mutation and a predicted loss-of-function variant were also identified in BMP10 in two severely affected sporadic PAH female patients.

Conclusion: Our results confirm that mutations are found in genes beyond BMPR2 in heritable PAH, emphasise the role of TBX4 and BMP9, and designate BMP10 as a new PAH gene. 


\section{Introduction}

Pulmonary arterial hypertension (PAH) is a rare and severe disease of the small pulmonary arteries characterised by a progressive increase in pulmonary vascular resistance leading to right ventricular failure and ultimately death in the absence of treatment or transplantation. A diagnosis of idiopathic PAH (IPAH) is made when no known risk factor or associated disease is authenticated [1]. In addition to idiopathic forms, heritable forms of the disease represent $\sim 30 \%$ of PAH cases (heritable PAH (HPAH)), and this category includes familial forms (familial PAH (FPAH)), with or without identified mutations, and sporadic forms carrying a mutation [1]. HPAH is a genetically heterogeneous disease that is transmitted as an autosomal dominant disease with incomplete penetrance. The major gene whose mutations are involved in HPAH is BMPR2 (bone morphogenetic protein receptor type 2), a gene encoding a type 2 receptor of the BMP signalling pathway [2]. BMPR2 mutations are found in $>80 \%$ of FPAH and in $\sim 15 \%$ of sporadic PAH $[3,4]$. BMPR 2 mutation carriers will develop the disease in $\sim 14 \%$ of males and $\sim 42 \%$ of females, although this proportion varies widely from family to family [5]. Mutations in other genes of the same pathway, ACVRL1 (activin A receptor-like type 1) and in rare cases ENG (endoglin), may cause PAH associated with hereditary haemorrhagic telangiectasia (HHT) [6, 7]. Mutations of SMAD9, encoding transcription factor Smad8, an important downstream mediator of BMP signalling, and in BMP9 (alias GDF2 (growth and differentiation factor 2)), encoding the ligand of ACVRL1, have been described in PAH $[8,9]$. Mutations in other genes, not related to the BMP signalling pathway, have also been identified. Mutations in KCNK3 (potassium channel two-pore domain subfamily $\mathrm{K}$ member 3; alias TASK1 (TWIK-related acid-sensitive $\mathrm{K}^{+}$channel 1)), encoding a voltage-sensitive potassium channel, have been found in a few families with $\mathrm{PAH}$ and the functional consequences of identified missense mutations were demonstrated in vitro [10]. A homozygous KCNK3 loss-of-function (LOF) variant was subsequently described in a paediatric case of severe $\mathrm{PAH}$ in a consanguineous family [11]. Only three mutations in the CAV1 gene encoding caveolin-1 have been identified so far: one in a large family with multiple cases of $\mathrm{PAH}$, another one as a de novo mutation in a young child and the third was associated with congenital generalised lipodystrophy [12, 13].

TBX4 (T-box 4) mutations have been recognised at the origin of PAH, through overlapping chromosomal deletions encompassing TBX4 and inducing $\mathrm{PAH}$ in children, together with bone anomalies, that prompted investigators to search for mutations in the TBX4 gene in other cases of paediatric PAH [14]. TBX4 heterozygous inactivating mutations are responsible for the small patella syndrome (also called coxopodopatellar syndrome) that associates bone anomalies of the patella, pelvis, foot and femur at variable degrees. It was previously reported that the proportion of patients carrying a TBX4 mutation was high in paediatric cases and higher than in adults, suggesting a specific impact of mutations of this gene in children $[15,16]$.

Pulmonary veno-occlusive disease (PVOD) and pulmonary capillary haemangiomatosis (PCH) are both characterised by diffuse remodelling of venules and septal veins by intimal fibrosis associated with patchy capillary proliferation and various degrees of arterial lesions. Biallelic mutations of EIF2AK4 (eukaryotic translation initiation factor $2 \alpha$ kinase 4 ) have been shown to be responsible for the heritable forms of PVOD and PCH that are transmitted as a recessive disease $[17,18]$. Heritable forms of PVOD and PCH (both further referred to here as PVOD) have the same clinical presentation with a poor prognosis, and are considered as the same entity even if histological pulmonary lesions may differ (mainly by the importance of capillary proliferation).

Affiliations: ${ }^{1}$ Département de Génétique, Hôpital Pitié-Salpêtrière, AP-HP, Paris, France. ${ }^{2}$ UMR_S1166, Sorbonne Université, INSERM, and Institute for Cardiometabolism and Nutrition (ICAN). Paris, France. ${ }^{3}$ Université Paris-Sud, Faculté de Médecine, Université Paris-Saclay, Service de Pneumologie, Centre de Référence de l'Hypertension Pulmonaire, INSERM UMR_S999, Hôpital de Bicêtre, AP-HP, Le Kremlin-Bicêtre, France. ${ }^{4}$ M3C-Cardiologie Pédiatrique, Hôpital Necker-Enfants Malades, AP-HP, Paris, France. ${ }^{5}$ PhyMedExp, University of Montpellier, INSERM, CNRS, Montpellier, France. ${ }^{6}$ Département de Pneumologie et Addictologie, CHU Montpellier, Montpellier, France. ${ }^{7}$ Service de Cardiologie Maladies Vasculaires, CHU Gabriel Montpied, Clermont-Ferrand, France. ${ }^{8}$ Département de Pneumologie, CHRU Nancy, Université de Lorraine, INSERM U1116, Nancy, France. 'Service de Pneumologie, Centre National de Référence des Maladies Pulmonaires Rares, Hôpital Louis Pradel, Université Claude Bernard Lyon 1, UMR754, Lyon, France. ${ }^{10}$ Service de Pneumologie, Hôpital Pasteur, CHU Nice, Nice, France. ${ }^{11}$ Service de Pneumologie, Hôpital Larrey, Toulouse, France. ${ }^{12}$ Service de Pneumologie, CHU Nord de Marseille, AP-HM, Marseille, France. ${ }^{13}$ Service de Pneumologie, CHU de Bordeaux Hôpital Haut-Levêque, Pessac, France. ${ }^{1}{ }^{4}$ Service de Cardiologie Infantile et Congénitale, CHRU Lille-Hôpital Cardiologique, Lille, France. ${ }^{15}$ Département de Génétique Médicale, $\mathrm{CHU}$ la Timone Enfants, AP-HM, Marseille, France.

Correspondence: Florent Soubrier, UMR_S1166, Sorbonne Université, site Pitié-Salpêtrière, 91 Boulevard de l'Hôpital, 75013 Paris, France. E-mail: florent.soubrierdupmc.fr 
The goal of this study is to describe mutations that can be found in a prospectively collected series of patients with sporadic or FPAH or PVOD, and to infer the respective place of each known responsible gene in adult and paediatric forms of pulmonary hypertension. We report the genotype and phenotype characteristics of a large series of patients with PAH or PVOD explored by gene panel sequencing allowing detection of both single nucleotide variants (SNVs) and copy number variants (CNVs).

\section{Patients and methods Patients}

This study included 263 patients followed within the French pulmonary hypertension investigating centres, which include the French Referral Centre for Pulmonary Hypertension (Hôpital Bicêtre, Université Paris-Sud, Le Kremlin-Bicêtre, France) and associated expert centres. All cases had a clinical diagnosis of sporadic or FPAH, drug- and toxin-induced PAH, or sporadic PVOD established by their expert centre. The diagnosis of pre-capillary pulmonary hypertension was defined by haemodynamic measurement during right heart catheterisation of all patients included in the study, according to previously described protocols [19]. Sporadic cases of PAH patients were further referred to as IPAH after clinical, biological and genetic investigations eliminated all known causes [20]. Cases were considered as FPAH when at least two cases were reported in the family extending up to third-degree relatives. Patients diagnosed as associated PAH following hospital investigation (notably for connective tissue disease and HIV infection) were not included.

Age at diagnosis $<18$ years defines paediatric cases.

Diagnosis of confirmed or highly probable PVOD was established according to previously described criteria $[17,21]$ and, due to the overlapping inclusion period, a few PVOD patients were included in a previous publication [22]. All PVOD patients are sporadic cases in this study.

Clinical characteristics at PAH diagnosis were stored in the French Pulmonary Hypertension Registry for those included. This Registry was set up in agreement with French bioethics laws (Commission Nationale de l'Informatique et des Libertés) and patients gave their consent to be included [23]. More than $90 \%$ of patients included are of Caucasian European descent. All patients included in this study underwent genetic counselling and signed written informed consent.

All cases included in this study are prospectively collected incident index cases of pulmonary hypertension from 2014 to 2017 and DNA sequencing analysis was performed at diagnosis within the period of inclusion, except for eight previously negative patients for BMPR2 and ACVRL1 that were referred for de novo analysis with the gene panel.

\section{Capture design}

We designed a custom gene panel by targeting all exons, including $50 \mathrm{bp}$ of the flanking intronic sequence and $1 \mathrm{~kb}$ areas upstream and downstream of each gene of interest. This panel included nine genes analysed for molecular diagnosis of pulmonary hypertension (BMPR2, TBX4, EIF2AK4, CAV1, KCNK3, SMAD9, $A C V R L 1, E N G$ and BMP9). The BMP10 gene, a BMP9 paralogue, was also included in the capture design. This next-generation sequencing (NGS) panel also includes additional genes related to pulmonary hypertension and other hereditary vascular diseases (HHT, capillary and arteriovenous malformation) with a goal of clinical diagnosis and research, and accounting for $79 \%$ of the total target sequence.

\section{Target capture sequencing method}

Libraries were prepared using $1 \mu \mathrm{g}$ genomic DNA according to the KAPA sample preparation protocol (KAPA Biosystems, Wilmington, MA, USA). After appropriate quality controls, libraries were pooled (pool of 12 samples) and captured using the SeqCap EZ Choice Library (Roche/NimbleGen, Madison, WI, USA) following the manufacturer's protocol. Two pools of captured libraries ( 24 samples) were sequenced to generate $2 \times 150$ bp paired-end reads using the MiSeq system (Illumina, San Diego, CA, USA).

\section{Data analysis}

Quality control, processing and detection of variants were performed as a service provision by GenoDiag (Paris, France). Briefly, FASTQ sequence files were mapped to the human reference genome (hg19) with BWA version 7.12 (http://bio-bwa.sourceforge.net) and converted to BAM files with SAMtools version 1.1 (www.htslib.org). SNV and insertion/deletion (indel) calling was performed with GATK version 3.5 (https://software.broadinstitute.org/gatk). CNVs were detected by GenoDiag proprietary scripts. The detection is based on the coverage of each exon. A normalisation is applied in order to remove coverage bias according to variation between samples. The normalised coverage is then used to compute a $\mathrm{z}$-score. Two additional parameters are computed, i.e. the coverage dispersion and coverage ratio, that measure the sequencing quality and the deletion or duplication significance. These three values are used to filter the 
false-positive results and set the events confidence level. Each base must be covered by at least 40 reads to be validated. This method was validated on 23 samples harbouring SNVs or CNVs on the major genes tested (BMPR2, EIF2AK4 and ACVRL1).

The putative functional impact of missense variants was assessed in silico with SIFT, PolyPhen-2, Combined Annotation Dependent Depletion (CADD) Phred score and allele frequency in the Genome Aggregation Database gnomAD [24-26]. All databases and software were queried through Alamut version 2.7.1 (www.interactive-biosoftware.com/alamut-visual) except for the CADD Phred score that was obtained from the variant effect predictor tool at the Ensembl website (http://grch37.ensembl.org/Homo_sapiens/ Tools/VEP? $\mathrm{db}=$ core). Each variant was classified according to American College of Medical Genetics and Genomics (ACMG) criteria [27]. Any potential pathogenic variant was confirmed by Sanger sequencing for point mutations and multiplex ligation-dependent probe amplification (MLPA) when available (MRC-Holland, Amsterdam, The Netherlands) or quantitative PCR (qPCR) analysis for large rearrangements.

\section{Statistical analysis}

Data are presented as mean with standard deviation. Since the data did not follow a normal distribution, comparisons were performed using the nonparametric Mann-Whitney test or Fisher exact analysis when appropriate using XLStat 2014 (www.xlstat.com). The significance threshold $(\mathrm{p}<0.05)$ was corrected for multiple testing using the Benjamini-Hochberg correction.

In order to evaluate the association of $B M P 10$ variants with $\mathrm{PAH}$, we compared the frequency of $B M P 10$ missense variants with a probably damaging effect predicted by PolyPhen-2, a deleterious effect by SIFT and a CADD score $>20 \quad(n=32)$, and LOF variants $(n=3)$ reported in gnomAD with the frequency of $B M P 10$ variants with similar characteristics detected in our cohort $(n=2)$. Statistical comparisons were performed using Chi-squared analysis.

\section{Results}

Sequence analysis and quality controls

A capture panel was designed to enrich target DNA, which consisted of $150071 \mathrm{bp}$ covering 289 target captured regions. Our design and sequencing protocols were validated by screening a cohort of 23 patients that were previously analysed by Sanger sequencing and MLPA. All previously identified mutations $(\mathrm{n}=16)$ (SNVs and indels) and CNVs $(\mathrm{n}=4)$ were detected by NGS analysis. Reciprocally, the absence of mutation detection was confirmed in three patients.

A total of 29 different sequencing runs were analysed in this study. The mean \pm SD Q30 quality score was $91.5 \pm 3.8 \%$ for all runs. The mean coverage was $1106 \pm 353$ times for all analysed samples. $95.6 \pm 3.4 \%$ of reads mapped in regions of interest, $2.6 \pm 2.2 \%$ of reads mapped outside regions of interest and $1.8 \pm 1.6 \%$ were not mapped to the human genome.

\section{Mutations in tested pulmonary hypertension genes}

Following pedigree analysis and clinical examination, 180 patients were initially classified as sporadic PAH, 11 as FPAH (only index cases included), 13 as drug- and toxin-induced PAH, and 59 as sporadic PVOD (figure 1). A mutation was identified in 49 out of the 263 analysed patients. Identification of biallelic EIF2AK4 mutations in one initially suspected PAH case led us to reclassify this patient as PVOD. Thus, 35 mutations were identified in sporadic PAH patients (19.5\% of sporadic PAH), six in FPAH patients (54.5\% of FPAH) and eight in PVOD patients (13.3\% of PVOD) (table 1).

31 BMPR2 mutations (25 sporadic PAH and six FPAH), five TBX4 mutations (five sporadic PAH), two ACVRL1 (two sporadic PAH), two BMP9 (two sporadic IPAH), one SMAD9 (one sporadic PAH) and eight biallelic EIF2AK4 mutations (eight PVOD) were identified (table 1). Most of the BMPR2 mutations are truncating mutations, i.e. stop codons $(n=11)$ or indels $(n=1)$ disrupting the function of the gene (supplementary table S1). Three distinct missense mutations were identified: two were previously described and the third (p.A373V) is new. This mutation is located in the kinase domain of BMPR2, alters a conserved residue and is predicted to be deleterious by in silico tools (see Data analysis). According to ACMG criteria [27], this mutation is classified as likely pathogenic. Three splice mutations were found: two affecting a consensus splicing site and one located at position c.968-12 within intron 7 that is predicted to affect splicing following in silico analysis. Reverse transcription PCR experiments using RNA extracted from circulating blood cells from the mutation carrier confirmed that mRNA splicing was defective, leading to the addition of an intronic sequence into the mature mRNA and creating a frameshift (supplementary figure S1).

The remaining six BMPR2 mutations are CNVs that were detected by NGS and were confirmed by MLPA. Two other CNVs were also detected: one in SMAD9 and one in TBX4. Both were confirmed by qPCR 


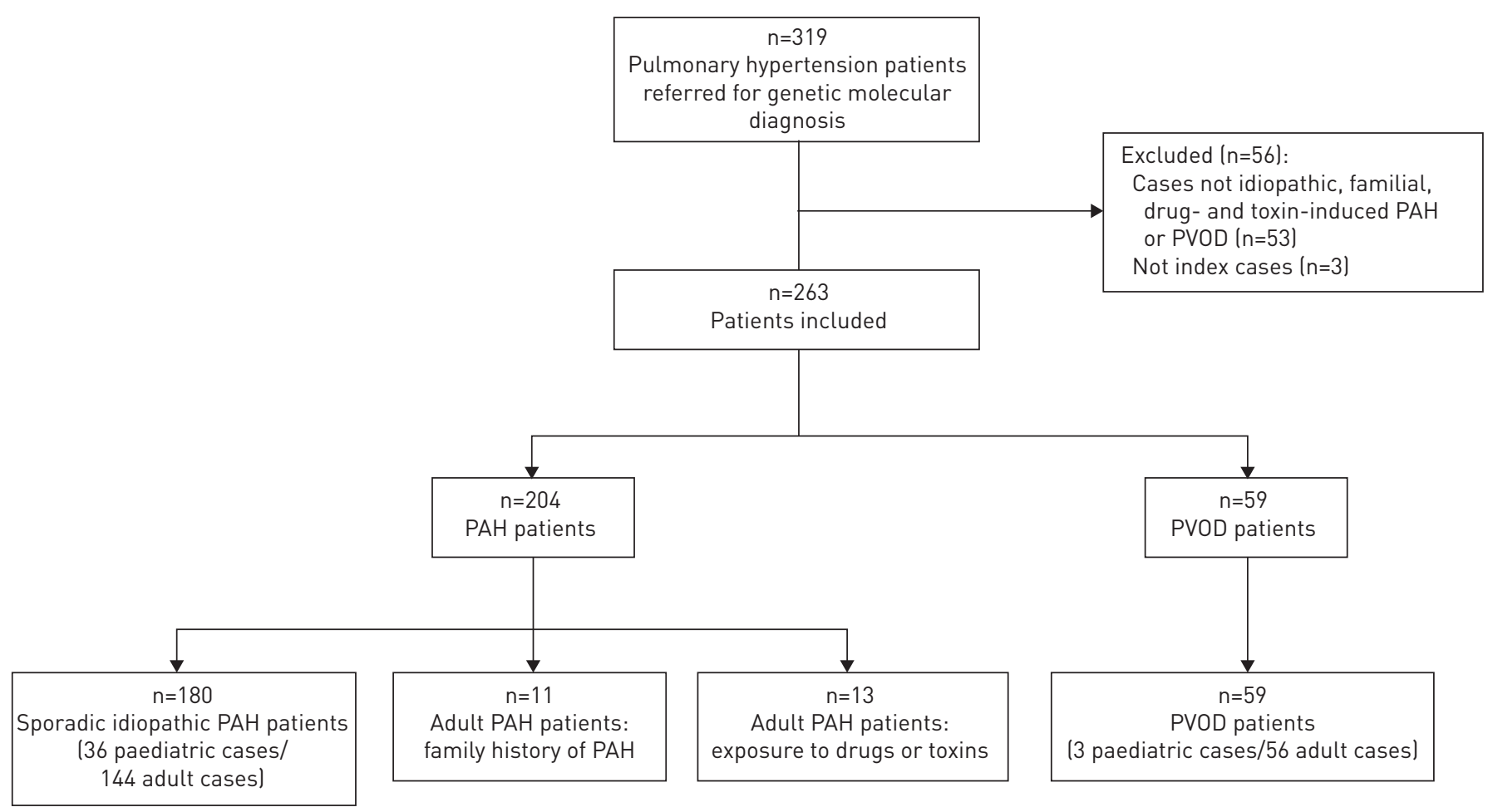

FIGURE 1 Flowchart of patients analysed in this study. PAH: pulmonary arterial hypertension; PVOD: pulmonary veno-occlusive disease. Diagnoses indicated are those provided with the molecular diagnosis request.

analysis. All EIF2AK4 mutations identified were biallelic: five at the homozygous state and three as compound heterozygotes.

Only truncating mutations were identified in TBX4 and BMP9 (stop codon $\mathrm{n}=2$, indel $\mathrm{n}=4$ and $\mathrm{CNV}$ $\mathrm{n}=1$ ), whereas two previously described missense mutations were identified in ACVRL1. HHT symptoms were present in the adult case of ACVRL1 mutation carriers, whereas the paediatric case had no sign of $\mathrm{HHT}$ at diagnosis and was referred to the laboratory as sporadic PAH.

The BMP10 gene was also included in the gene panel, after the description of BMP9 mutations in PAH and because of the close similarity between the two genes (65\% sequence identity at the protein level). A truncating mutation (c.370C>T, p.Arg $124^{*}$ ) located upstream of the region encoding the mature BMP10

TABLE 1 Mutations and variants of unknown significance (VUS) identified in established pulmonary arterial hypertension (PAH) and pulmonary veno-occlusive disease (PVOD) genes

\begin{tabular}{|c|c|c|c|c|c|c|}
\hline & \multicolumn{2}{|c|}{ Sporadic PAH $^{\#}$} & \multicolumn{2}{|c|}{ Familial PAH } & \multicolumn{2}{|c|}{ PVOD $^{+}$} \\
\hline & Mutation & VUS & Mutation & vUS & Mutation & VUS \\
\hline BMPR2 & 25 & 4 & 6 & 0 & 0 & 1 \\
\hline TBX4 & 5 & 1 & 0 & 0 & 0 & 0 \\
\hline ACVRL1 & 2 & 0 & 0 & 0 & 0 & 0 \\
\hline EIF2AK4 & 0 & 3 & 0 & 0 & $8^{\S}$ & 0 \\
\hline BMP9 & 2 & 1 & 0 & 0 & 0 & 0 \\
\hline SMAD9 & 1 & 1 & 0 & 0 & 0 & 0 \\
\hline KCNK3 & 0 & 1 & 0 & 0 & 0 & 0 \\
\hline CAV1 & 0 & 1 & 0 & 0 & 0 & 0 \\
\hline ENG & 0 & 1 & 0 & 0 & 0 & 0 \\
\hline Total & 35 (19.5) & 13 (7.3) & $6(54.5)$ & 0 & 8 (13.3) & $1(1.7)$ \\
\hline
\end{tabular}

Data are presented as $\mathrm{n}$ or $\mathrm{n}(\%)$. One paediatric case carrying EIF2AK4 biallelic mutations was shifted from PAH to PVOD. Drug- and toxin-induced PAH patients are excluded since no mutation was found in this category of patients. ${ }^{\#}: \mathrm{n}=179 ;{ }^{\uparrow}: \mathrm{n}=11 ;{ }^{+}: \mathrm{n}=60 ;{ }^{\S}$ : all EIF2AK4 mutations are biallelic mutations. 
TABLE 2 Clinical, functional and haemodynamic characteristics of patients carrying a BMP10 mutation

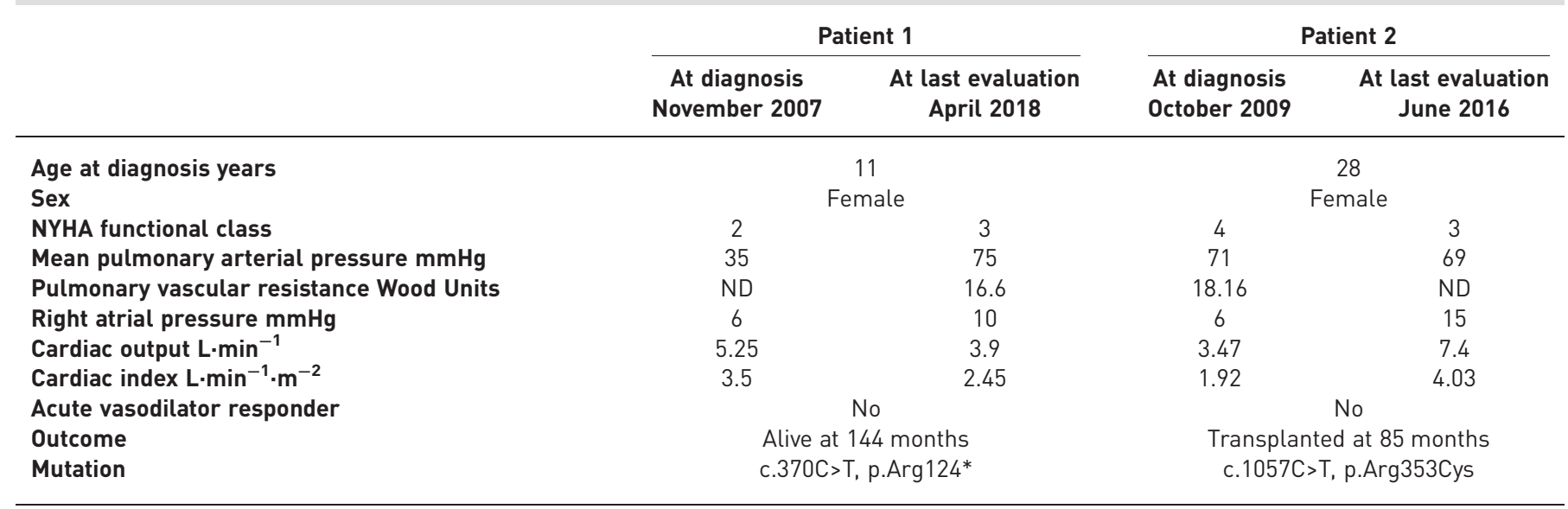

NYHA: New York Heart Association; ND: not determined.

peptide was found in a young female patient who was diagnosed with sporadic PAH at 11 years with severe evolution (table 2, patient 1). This young patient had a small atrial septal defect and a small restrictive ventricular septal defect that spontaneously closed at 2 years of age. A predicted BMP10 LOF mutation (c.1057C > T, p.Arg353Cys) was identified in another patient who was diagnosed with sporadic $\mathrm{PAH}$ at age 28 years in a severe clinical condition and transplanted at age 35 years (table 2, patient 2). Arginine 353 is located in the growth factor fragment of BMP10, is highly conserved between species (supplementary table S2) and the whole in silico analysis of this variant is consistent with a deleterious effect (table 4). The probability of being LOF intolerant was calculated for BMP10 and was found to be 0.72, a value close to the intolerance threshold of 0.9 at which LOF mutations are likely deleterious, compared with a value of 0.09 for BMP9 [24]. A highly significant difference was found between the frequency of probably deleterious variants according to combined bioinformatic predicting tools (see Data analysis) and truncating mutations of BMP10 listed in the gnomAD database and the frequency of LOF in our pulmonary hypertension cohort (35 different variants on 40 alleles among 246000 alleles versus two LOF variants among 536 tested alleles; $\mathrm{p}<0.0001)$.

Patients referred presented with various ages at diagnosis, ranging from neonates to elderly adults ( $>80$ years). In order to get an accurate insight into the genetic landscape of mutations found in adult and paediatric cases, a separate analysis was performed in patients under and above 18 years of age (table 3). 35 paediatric and 168 adult PAH cases were analysed. No significant difference was observed in the mutation rate between adult and paediatric PAH cases (19\% and 26\%, respectively). BMPR2 mutations are the most frequent in both age groups $(75 \%$ and $77 \%$ of identified mutations in adult and paediatric groups, respectively). TBX4 mutations are detected with a similar frequency in adult and paediatric cases ( $12.5 \%$ and $11 \%$ of identified mutations in each group, respectively).

In one case referred clinically as neonatal PAH, a biallelic mutation of EIF2AK4 was found, leading to a reclassification as unrecognised PVOD. In contrast, no mutation was found in the three other paediatric cases initially diagnosed as PVOD. In adult PVOD patients, only biallelic EIF2AK4 mutations were identified (12.5\% of cases tested positive).

\section{Variants of uncertain significance identified}

According to ACMG criteria [27], 14 variants of uncertain significance (VUS) were found across the genes investigated. In silico analysis of VUS is provided in table 4. No pathogenic mutation was identified in KCNK3 and CAV1, but two VUS were identified in these genes. The missense variant of KCNK3 was found to be located within an extracellular domain of the channel, a location similar to the missense mutations previously shown to alter the function of the channel in the first study involving this gene in PAH. An in-frame deletion of a single amino acid was identified in CAV1 and should also be considered as a VUS.

Two BMPR2 promoter variants, not described in the gnomAD database, were identified. Two missense EIF2AK4 variants were identified in the same individual, but their locations on the two different alleles could not be determined. 
TABLE 3 Mutations identified in paediatric and adult cases

\begin{tabular}{|c|c|c|c|c|}
\hline & \multicolumn{2}{|c|}{ PAH } & \multicolumn{2}{|c|}{ PVOD } \\
\hline & Paediatric cases & Adult cases & Paediatric cases & Adult cases \\
\hline Subjects & 35 & 168 & 4 & 56 \\
\hline Age at diagnosis years & $6.6 \pm 5.3(0.03-16)$ & $51.8 \pm 17.8(18-86)$ & $8.8 \pm 6.1(0.01-13)$ & $57.7 \pm 15.8(21-88)$ \\
\hline Female/male & $21(60) / 14(40)$ & $117(70) / 51(30)$ & $1(25) / 3(75)$ & $22(39) / 34(60)^{\#}$ \\
\hline BMPR2 & 7 & 24 & 0 & 0 \\
\hline EIF2AK4 & 0 & 0 & 1 & 7 \\
\hline TBX4 & 1 & 4 & 0 & 0 \\
\hline ACVRL1 & 1 & 1 & 0 & 0 \\
\hline BMP9 & 0 & 2 & 0 & 0 \\
\hline SMAD9 & 0 & 1 & 0 & 0 \\
\hline Total & $9(26)$ & 32 (19) & $1(25)$ & $7(12.5)$ \\
\hline
\end{tabular}

Data are presented as $n$, mean \pm SD (minimum-maximum) or $n(\%)$. PAH: pulmonary arterial hypertension; PVOD: pulmonary veno-occlusive disease. One paediatric case carrying EIF2AK4 biallelic mutations was shifted from PAH to PVOD. Comparisons between sex ratio in adult PAH and adult PVOD were analysed by the Fisher exact test. ${ }^{\#}: \mathrm{p}<0.0001$.

\section{Clinical status at time of diagnosis}

Comparison of the clinical presentation at diagnosis between the mutation carrier and noncarrier groups is shown in table 5 for adult PAH cases, paediatric PAH cases and adult PVOD cases. The age at diagnosis is significantly lower in the mutation carriers than in noncarriers in both adult PAH and PVOD, but not in paediatric PAH. The sex distribution observed in adult PAH (2- to 3-fold more females) is similar to that previously described in various cohorts analysed $[5,28]$. A similar tendency was observed in PAH paediatric cases. The sex distribution shows a nonsignificant predominance of males in noncarrier PVOD cases, a result contrasting with PAH ( $\mathrm{p}<0.0001$; table 3), and no difference in EIF2AK4 mutation carriers (tables 3 and 5).

The haemodynamic parameters tended to be worse in mutation carriers even if only mean pulmonary arterial pressure in adult $\mathrm{PAH}$ reached statistical significance.

The proportion of acute vasodilator responders tends to be higher in noncarriers in both adult and paediatric PAH cases even if statistical significance is not reached (table 5).

\section{Discussion}

Massively parallel sequencing (i.e. NGS) has revolutionised the molecular genetics of hereditary diseases, allowing new causative genes to be identified and allowing a huge increase in clinical molecular diagnosis

TABLE 4 Analysis of variants of unknown significance (VUS) identified

\begin{tabular}{|c|c|c|c|c|c|c|}
\hline & HGVS coding DNA & HGVS protein & gnomAD MAF \% & PolyPhen-2 & SIFT & CADD Phred score \\
\hline BMPR2 & c. $746 \mathrm{~T}>\mathrm{G}$ & p.(Val249Gly) & 0.00072 & Possibly damaging & Deleterious & 26.3 \\
\hline BMPR2 & c. $1981 \mathrm{G}>\mathrm{C}$ & p.(Glu661Gln) & 0.00081 & Probably damaging & Deleterious & 26 \\
\hline BMPR2 & c. $-450 C>G$ & p.? & $N F$ & NA & NA & NA \\
\hline BMPR2 & c. $-816 A>G$ & p.? & NF & NA & NA & NA \\
\hline EIF2AK4 & c. $179 A>G$ & p.(Tyr60Cys) & NF & Possibly damaging & Deleterious & 24.1 \\
\hline TBX4 & c. $1021 \mathrm{G}>\mathrm{C}$ & p.(Asp341His) & NF & Possibly damaging & Deleterious & 24.2 \\
\hline KCNK3 & c. $253 T>C$ & p.(Tyr85His) & $N F$ & Probably damaging & Deleterious & 26.5 \\
\hline SMAD9 & c. $283 \mathrm{G}>\mathrm{A}$ & p.(Val95Met) & $\mathrm{NF}$ & Probably damaging & Tolerated & 23.7 \\
\hline ENG & c. $821 C>T$ & p.(Thr274Ile) & 0.00041 & Probably damaging & Deleterious & 26.5 \\
\hline BMP9 & c. $427 G>A$ & p.(Glu143Lys) & NF & Possibly damaging & Deleterious & 28 \\
\hline CAV1 & c.336_338del & p.(Leu113del) & NF & NA & NA & NA \\
\hline
\end{tabular}

HGVS: Human Genome Variation Society; MAF: minor allele frequency; CADD: Combined Annotation Dependent Depletion; NF: not found; NA: not applicable. " : CADD Phred score $\geqslant 20$ indicates the $1 \%$ most deleterious variants on the genome sequence [25]; " : two EIF2AK4 variants were found in the same individual (but haplotypes are not defined). 
TABLE 5 Demographics and clinical measurements at diagnosis: adult pulmonary arterial hypertension (PAH) cases, paediatric PAH cases and adult pulmonary veno-occlusive disease (PVOD) cases

\section{Noncarriers}

\section{Adult PAH}

Subjects

Age at diagnosis years $(n=168)$

Minimum-maximum

Female/male

NYHA functional class $(n=156)$

I/II

III/IV

Mean pulmonary arterial pressure $\mathrm{mmHg}(n=156)$

Pulmonary vascular resistance Wood Units ( $n=146)$

Right atrial pressure $\mathrm{mmHg}(\mathrm{n}=151)$

Cardiac output L. $\mathrm{min}^{-1}(\mathrm{n}=155)$

Cardiac index $L \cdot \mathrm{min}^{-1} \cdot \mathrm{m}^{-2}(\mathrm{n}=153)$

Vasodilator responder $(n=112)$

\section{Paediatric PAH}

Subjects

Age at diagnosis years ( $n=35$ )

Minimum-maximum

Median (IQR)

Female/male

NYHA functional class ( $n=25$ )

I/II

III/IV

Mean pulmonary arterial pressure $\mathrm{mmHg}(\mathrm{n}=29)$

Median (IQR)

Pulmonary vascular resistance Wood Units ( $n=27$ )

Median (IQR)

Right atrial pressure $\mathrm{mmHg}(\mathrm{n}=25)$

Median (IQR)

Cardiac output L. $\min ^{-1}(\mathrm{n}=25)$

Median (IQR)

Cardiac index $\mathrm{L} \cdot \mathrm{min}^{-1} \cdot \mathrm{m}^{-2}(\mathrm{n}=24)$

Median (IQR)

Acute vasodilator responder $(n=26)$

\section{Adult PVOD}

Subjects

Age at diagnosis years ( $n=56$ )

Minimum-maximum

Median (IQR)

Female/male

NYHA functional class ( $n=47)$

I/II

III/IV

Mean pulmonary arterial pressure $\mathrm{mmHg}(\mathrm{n}=48)$

Median (IQR)

Pulmonary vascular resistance Wood Units ( $n=46$ )

Median (IQR)

Right atrial pressure $\mathrm{mmHg}(\mathrm{n}=45)$

Median (IQR)

Cardiac output L. $\min ^{-1}(\mathrm{n}=46)$

Median (IQR)

Cardiac index $\mathrm{L} \cdot \mathrm{min}^{-1} \cdot \mathrm{m}^{-2}(\mathrm{n}=47)$

Median (IQR)

Solvent exposure or previous chemotherapy $(n=40)$

136
$54.0 \pm 18$
$18-86$
$93(68) / 43(32)$
$33(26)$
$94(74)$
$50.2 \pm 13.5$
$11.6 \pm 6.2$
$8.3 \pm 5.2$
$4.2 \pm 1.5$
$2.3 \pm 0.8$
$12(13.5)$

26
$5.4 \pm 5.3$
$(0.035-16)$
$4.1(6.9)$
$15(58) / 11(42)$

$7(41)$

10 (59)

$51 \pm 17.7$

51 (23)

$22.3 \pm 18.6$

17.3 (17.3)

$6.2 \pm 3.4$

5.5 (4)

$2.6 \pm 1.2$

2.5 (1.4)

$3.8 \pm 1.3$

$3.3(1.4)$

6 (33)

49
$61 \pm 13.2$
$22-88$
$64(13)$

$19(39) / 30(61)$

$$
\begin{gathered}
11(25) \\
33(75) \\
47.3 \pm 11.8 \\
47.5(17.2) \\
10.2 \pm 9.4 \\
8.7(4.3) \\
8 \pm 5.1 \\
7(6) \\
4.4 \pm 1.1 \\
4.5(1.3) \\
2.4 \pm 0.6 \\
2.4(0.8) \\
17(42.5)
\end{gathered}
$$

\section{Carriers}

p-value

$\begin{array}{cc}32^{\#} & \\ 42.5 \pm 12.3 & <0.0001^{\S} \\ 20-69 & \\ 24(75) / 8(25) & 0.527 \\ 6(21) & 0.641 \\ 23(79) & \\ 57.4 \pm 13.1 & 0.004^{\S} \\ 14.4 \pm 7.3 & 0.036 \\ 9.6 \pm 6.2 & 0.385 \\ 3.8 \pm 1.4 & 0.056 \\ 2.2 \pm 0.9 & 0.086 \\ 1(4.3) & 0.297\end{array}$

9ी
$10 \pm 3.7$
$(4.5-15)$
$10.2(5.2)$
$6(67) / 3(33)$

0.020

0.712

5 (62)

3 (38)

$55.1 \pm 15.8$

54.5 (15.7)

$13.6 \pm 6.8$

$14(7.6)$

$5.3 \pm 1.8$

5 (1)

$4.5 \pm 3$

3.2 (1.3)

$3.6 \pm 1.2$

3.1 (1.1)

$1(12)$

0.494

0.300

0.807

0.066

0.854

0.375

$7^{+}$

$34.4 \pm 13.1$

$21-52$

35 (21.5)

$3(43) / 4$ (57)

2 (67)

$47.7 \pm 20.3$

$38.5(14.2)$

$9.9 \pm 6.2$

$7.2(4.4)$

$5.2 \pm 2.1$

5.5 (3.2)

$3.8 \pm 0.4$

$3.8(0.5)$

$2.3 \pm 0.2$

$2.2(0.2)$

0 (0)
$<0.0001^{\S}$

1

0.181

0.614

0.770

0.336

0.250

0.542

0.014

Data are presented as $\mathrm{n}$, mean \pm SD or $\mathrm{n}(\%)$, unless otherwise stated (e.g. median (interquartile range (IQR)) for small subgroups). NYHA: New York Heart Association. Comparisons between mutation carriers and noncarriers were analysed by Mann-Whitney or Fisher exact tests. ${ }^{\#}$ : EIF2AK4 mutation carriers excluded (the unique acute vasodilator responder carrier has a BMPR2 mutation c.1471C>T, p.Arg491Trp); ๆ: EIF2AK4 mutation carriers excluded (the unique vasodilator responder carrier has a TBX4 mutation c.143dup, p.Pro50Thrfs*24); ${ }^{+}$: EIF2AK4 mutations carriers; ${ }^{\S}$ : significant $p$-value after Benjamini-Hochberg correction for multiple testing. 
efficiency [29]. Targeted NGS on a panel of recognised genes for the disease is an efficient approach, allowing an extensive, economically acceptable, investigation of the potentially responsible genes.

In this prospective study of incident cases, we have used the NGS panel approach in patients referred to a reference laboratory for PAH or PVOD. The panel of genes included established genes through previous publications and research genes based on physiological functions and previous data available. Using this NGS targeted capture approach, the diverse types of mutations (SNVs, CNVs and indels) could be detected by a single technique allowing cost and time savings.

BMPR2 is the predominant gene in adult (familial and sporadic) as in paediatric forms of PAH. In FPAH cases, only mutations in BMPR2 were found, possibly because of its predominant frequency, but more specific reasons might be involved linked to penetrance and clinical evolutions associated to the mutated gene. These results are in accordance with previously published results [30, 31].

In the present study, TBX4 mutations were the second most frequent mutations found after BMPR2 in both adult and paediatric PAH cases. We previously observed a frequent occurrence of TBX4 mutations in paediatric PAH [15], a result recently corroborated by another genetic study [16]. The significant frequency of TBX4 mutations found in adult cases in this study, contrasting with previously reported results [16], could be due to stochastic differences as well as to differences in the mode of patient collection (prevalent and archived patients versus incident cases).

Mutations in other genes of the BMP signalling pathway were identified (BMP9 and SMAD9) in a small number of cases, allowing the implication of these genes in PAH to be confirmed since only rare cases were described in the literature. Indeed, the initial description of BMP9 mutations was associated with a particular form of HHT and followed by a description of a case of severe PAH associated with biallelic LOF $[32,33]$. Rare heterozygous BMP9 mutations were recently described in a large group of patients explored by whole-genome sequencing [9]. In our study, BMP9 is the third most frequently mutated gene in adult $\mathrm{PAH}$ cases (1.2\% of $\mathrm{PAH}$ cases, $6.3 \%$ of identified mutations), suggesting that this gene has a significant relevance in PAH. A mouse genetic model with heterozygous BMPR2 LOF shows an improvement of hypoxia-induced $\mathrm{PAH}$ by BMP9 infusion, a result that further supports, although indirectly, the involvement of this gene in PAH [34]. Within the setup of a research endeavour, we investigated $B M P 10$, a close paralogue of BMP9 that encodes an activating ligand for ALK1 (ACVRL1) and that was shown to play an equivalent role as BMP9 in an experimental model of angiogenesis since it can substitute for BMP9 in BMP9 knockout mice $[35,36]$. It was also recently shown that the BMP9-BMP10 heterodimer circulates in human plasma and is the major biologically receptor-activating complex, although the additional function of this heterodimer is not identified [37]. We found a BMP10 heterozygous truncating mutation and an in silico predicted LOF missense variant in two cases of severe $\mathrm{PAH}$ in young female patients. One of the two patients presented with a small atrial left-to-right shunt and a small highly restrictive ventricular septal defect with no pulmonary overflow that spontaneously closed before 2 years of age, whereas PAH was diagnosed at age 11 years. This clinical presentation does not allow this case to be classified as a coincident case or a post-operative case according to the 2013 classification of PAH associated with congenital heart disease [1], but raises the question of the relationship between the BMP10 heterozygous LOF, heart development and $\mathrm{PAH}$, since Bmp10-deficient mice embryos die very early, between embryonic days 9.5 and 10.5 , with major defects in heart and vascular development [38, 39]. Identifying two LOF variants in BMP10 in this prospective cohort of patients is highly suggestive of its responsibility in $\mathrm{PAH}$, because of the physiological role of this gene, its score of probability of being LOF intolerant, and the highly significant difference of LOF variant frequency between patients and an unselected population. BMP10-associated predisposition to PAH seems compatible with an autosomal dominant transmission with incomplete penetrance, but might also act as a predisposing factor in combination with other factors. Indeed, some missense BMP10 variants, likely pathogenic according to bioinformatic predicting tools, are found in the gnomAD database at extremely low frequency, as also observed for other PAH genes. Further studies will allow the role of BMP10 mutations to be defined. No pathogenic mutation of CAV1 was found in this study, in agreement with previous reports and on large series of patients $[9,30]$, and altogether these results argue for a very limited involvement of this gene in PAH predisposition.

The rate of biallelic EIF2AK4 LOF mutations found in adult sporadic PVOD cases (12.5\%) is consistent with our previously published results on sporadic cases [22], but lower than in a reported series [39]. This discrepancy is likely due to random variation favoured by the limited number of cases and patient collection specificities. The only biallelic EIF2AK4 mutation found in paediatric forms of PVOD was in a neonatal case initially referred for $\mathrm{PAH}$ molecular diagnosis suggested by early cardiorespiratory failure [22]. This case emphasises the importance of EIF2AK4 testing in all putative cases in order to establish the diagnosis because the clinical picture can be misleading, and investigations that can best allow distinction between 
PAH and PVOD (high-resolution computed tomography and diffusing capacity of the lung for carbon monoxide) might be not clearly discriminating or missing, or even contraindicated (lung biopsy) [22, 30, 31]. EIF2AK4 mutation identification by clinical genetic testing is particularly important with regard to high penetrance and disease severity of hereditary PVOD, to the risk of pulmonary oedema in response to approved therapies for $\mathrm{PAH}$, and because it allows genetic counselling and adapted follow-up including timely referral for lung transplantation. EIF2AK4 mutation interpretation is usually straightforward but in one case, two missense EIF2AK4 mutations were found in a patient with PVOD, both considered in silico as benign and found at a significant frequency in the gnomAD database. It can be speculated that carrying both missense variants, if located in trans, might lead to the disease, but this hypothesis needs further investigation.

Age at onset and sex variations in the various patient categories referred for genetic analysis are the consequences of the mixture of heritable forms (for which penetrance is the major factor determining these parameters) and nonheritable forms (for which environmental factors are predominant). The sex ratio observed in $\mathrm{PAH}$ is in agreement with previous values found in various cohorts, both in mutation carriers and noncarriers [5]. In the adult EIF2AK4 mutation carrier group, the sex ratio analysis is limited by the number of subjects but seems equilibrated. Indeed, a strong penetrance of EIF2AK4 mutations in both sexes likely explains the equilibrated sex ratio, as expected in autosomal recessive diseases.

The age at diagnosis is also younger in the mutation carrier PAH group, as previously reported for BMPR2 mutation carriers [28]. In the adult PVOD group, there is a strong difference in the age of onset between mutation carriers and noncarriers, an observation already reported by our group [17, 22]. Well specified environmental factors have been previously documented in nonheritable PVOD and likely explain the large difference in age of onset between heritable forms and other forms (34.4 versus 61.2 years; $\mathrm{p}<0.0001)$.

Our data reporting the sequence analysis of the whole set of described genes to date still leave a high proportion of cases unresolved, in particular paediatric and familial cases. Identifying and interpreting the functional impact of all variants in known responsible genes, including in noncoding regions, remains a major practical challenge that requires further efforts and knowledge, but which would elucidate a significant number of cases [29]. For example, in our study, we identified a variant located in a nonconsensus splicing site (intronic position -12) and we demonstrated by cDNA analysis that it alters mRNA splicing. We also identified two VUS in the BMPR2 promoter, and functional studies are necessary to evaluate the pathological effect of these variants on gene regulation.

Missing parameters of functional data for a number of patients limit the impact of this study in terms of genotype-phenotype relationships, although this aspect is beyond the scope of the study.

Mutations in additional genes can be found by whole-exome sequencing and whole-genome sequencing of large series of unrelated cases or in families with multiple cases of pulmonary hypertension. Indeed, rare variants in previously undescribed genes were identified recently by the whole-genome sequencing approach in a large series of PAH cases [9] and by whole-exome sequencing in a search for de novo mutations in paediatric cases [16]. These newly identified genes can be easily added to targeted panels for further evaluation and clinical molecular diagnosis. For several reasons, economical and technical, the targeted capture panel approach is currently the most efficient as a first diagnostic step for clinical molecular diagnosis.

A major challenge will be to elucidate whether the various genes predispose to $\mathrm{PAH}$ or PVOD development through a common mechanism, as can be proposed for genes belonging to the BMP signalling pathway, or through different mechanisms. As an example, the BMP pathway is important for mesoderm and mesenchymal cell development during embryogenesis, as is also the case for TBX4 that drives mesenchymal progenitor invasion of the lung during development [40]. BMP4 was also shown to induce TBX4 expression in cultured human lung fibroblasts [41]. A regulatory connection between EIF2AK4 and BMP signalling pathways was recently described in Drosophila, but the observed increase in BMP signalling activation following Eif2ak4 Drosophila orthologue inactivation does not fit with the expected effects of BMPR2 deficiency [42].

An overarching analysis of our data on known pulmonary hypertension genes seems to confirm the involvement of all major genes previously described if not only LOF mutations but also missense variants that have been found on the various genes are taken into account. Indeed, imputing a pathogenic role in $\mathrm{PAH}$ to heterozygous mutations is not in doubt for major genes such as BMPR2 and TBX4, but may be more difficult for other genes, for which fewer data are available on PAH. One interest of our data is to spur the analysis of the BMP9 and BMP10 genes in clinical molecular diagnosis of PAH, allowing results from several centers or from large multicentric studies to be shared in the future. 
Acknowledgements: We thank Anne Leroy and Marie-Christine Waill (Département de Génétique, Hôpital PitiéSalpêtrière, AP-HP, Paris, France) for expert technical assistance. We thank the patients, their families and healthcare providers from the French Pulmonary Hypertension Network for agreeing to collaborate, and the patient association HTAP France.

Conflict of interest: M. Eyries reports personal fees from Roche Diagnostics France, during the conduct of the study. D. Montani reports personal fees from Actelion, GSK, MSD and Pfizer, outside the submitted work. S. Nadaud has nothing to disclose. B. Girerd has nothing to disclose. M. Levy has nothing to disclose. A. Bourdin reports grants, personal fees for speaking and nonfinancial support from GSK, grants, personal fees for speaking and advisory board work, and nonfinancial support from AstraZeneca and Boehringer Ingelheim, personal fees for speaking and advisory board work, and nonfinancial support from Novartis, grants from MSD, grants and personal fees for speaking from Actelion, and personal fees for speaking and nonfinancial support from Chiesi Farmaceuticals, outside the submitted work. R. Trésorier has nothing to disclose. A. Chaouat has nothing to disclose. V. Cottin reports personal fees for consultancy, lectures and travel to medical meetings from Actelion and Roche, personal fees for development of educational presentations, consultancy, lectures and travel to medical meetings from Boehringer Ingelheim, personal fees for consultancy from Bayer, personal fees for adjudication committee work from Gilead, personal fees for consultancy and travel to medical meetings from MSD, personal fees for consultancy and lectures from Novartis and Sanofi, institutional grants from Boehringer Ingelheim and Roche, personal fees for data and safety monitoring board work from Promedior and Celgene, and personal fees for consultancy and data and safety monitoring board work from Galapagos, outside the submitted work. C. Sanfiorenzo has nothing to disclose. G. Prevot has nothing to disclose. M. Reynaud-Gaubert has nothing to disclose. C. Dromer has nothing to disclose. A. Houeijeh has nothing to disclose. K. Nguyen has nothing to disclose. F. Coulet has nothing to disclose. D. Bonnet reports personal fees for advisory board work from Actelion Pharmaceuticals, Bayer Health Care and Novartis, outside the submitted work. M. Humbert reports personal fees from Actelion, Bayer, GSK, Johnson \& Johnson, Merck and United Therapeutics, outside the submitted work. F. Soubrier has nothing to disclose.

Support statement: This work by supported by the Legs Poix (Chancellerie des Universités de Paris). Funding information for this article has been deposited with the Crossref Funder Registry.

\section{References}

1 Simonneau G, Gatzoulis MA, Adatia I, et al. Updated clinical classification of pulmonary hypertension. J Am Coll Cardiol 2013; 62: D34-D41.

2 Lane KB, Machado RD, Pauciulo MW, et al. Heterozygous germline mutations in BMPR2, encoding a TGF-beta receptor, cause familial primary pulmonary hypertension. The International PPH Consortium. Nat Genet 2000; 26: 81-84.

3 Machado RD, Southgate L, Eichstaedt CA, et al. Pulmonary arterial hypertension: a current perspective on established and emerging molecular genetic defects. Hum Mutat 2015; 36: 1113-1127.

4 Girerd B, Montani D, Jaïs X, et al. Genetic counselling in a national referral centre for pulmonary hypertension. Eur Respir J 2016; 47: 541-552.

5 Larkin EK, Newman JH, Austin ED, et al. Longitudinal analysis casts doubt on the presence of genetic anticipation in heritable pulmonary arterial hypertension. Am J Respir Crit Care Med 2012; 186: 892-896.

6 Trembath RC, Thomson JR, Machado RD, et al. Clinical and molecular genetic features of pulmonary hypertension in patients with hereditary hemorrhagic telangiectasia. N Engl J Med 2001; 345: 325-334.

7 Chaouat A, Coulet F, Favre C, et al. Endoglin germline mutation in a patient with hereditary haemorrhagic telangiectasia and dexfenfluramine associated pulmonary arterial hypertension. Thorax 2004; 59: 446-448.

8 Shintani M, Yagi H, Nakayama T, et al. A new nonsense mutation of SMAD8 associated with pulmonary arterial hypertension. J Med Genet 2009; 46: 331-337.

9 Gräf S, Haimel M, Bleda $\mathrm{M}$, et al. Identification of rare sequence variation underlying heritable pulmonary arterial hypertension. Nat Commun 2018; 9: 1416.

10 Ma L, Roman-Campos D, Austin ED, et al. A novel channelopathy in pulmonary arterial hypertension. $N$ Engl Med 2013; 369: 351-361.

11 Navas Tejedor P, Tenorio Castaño J, Palomino Doza J, et al. An homozygous mutation in KCNK3 is associated with an aggressive form of hereditary pulmonary arterial hypertension. Clin Genet 2017; 91: 453-457.

12 Austin ED, Ma L, LeDuc C, et al. Whole exome sequencing to identify a novel gene (caveolin-1) associated with human pulmonary arterial hypertension. Circ Cardiovasc Genet 2012; 5: 336-343.

13 Han B, Copeland CA, Kawano Y, et al. Characterization of a caveolin-1 mutation associated with both pulmonary arterial hypertension and congenital generalized lipodystrophy. Traffic 2016; 17: 1297-1312.

14 Kerstjens-Frederikse WS, Bongers EMHF, Roofthooft MTR, et al. TBX4 mutations (small patella syndrome) are associated with childhood-onset pulmonary arterial hypertension. J Med Genet 2013; 50: 500-506.

15 Levy M, Eyries M, Szezepanski I, et al. Genetic analyses in a cohort of children with pulmonary hypertension. Eur Respir J 2016; 48: 1118-1126.

16 Zhu N, Gonzaga-Jauregui C, Welch CL, et al. Exome sequencing in children with pulmonary arterial hypertension demonstrates differences compared with adults. Circ Genomic Precis Med 2018; 11: e001887.

17 Eyries M, Montani D, Girerd B, et al. EIF2AK4 mutations cause pulmonary veno-occlusive disease, a recessive form of pulmonary hypertension. Nat Genet 2014; 46: 65-69.

18 Best DH, Sumner KL, Austin ED, et al. EIF2AK4 mutations in pulmonary capillary hemangiomatosis. Chest 2014; 145: $231-236$.

19 Sitbon O, Humbert M, Jais X, et al. Long-term response to calcium channel blockers in idiopathic pulmonary arterial hypertension. Circulation 2005; 111: 3105-3111.

20 Galiè N, Humbert M, Vachiery J-L, et al. 2015 ESC/ERS Guidelines for the diagnosis and treatment of pulmonary hypertension: The Joint Task Force for the Diagnosis and Treatment of Pulmonary Hypertension of the European Society of Cardiology (ESC) and the European Respiratory Society (ERS): Endorsed by: Association for European Paediatric and Congenital Cardiology (AEPC), International Society for Heart and Lung Transplantation (ISHLT). Eur Respir J 2015; 46: 903-975. 
28 Girerd B, Montani D, Coulet F, et al. Clinical outcomes of pulmonary arterial hypertension in patients carrying an ACVRL1 (ALK1) mutation. Am J Respir Crit Care Med 2010; 181: 851-861.

29 Chakravorty S, Hegde M. Gene and variant annotation for Mendelian disorders in the era of advanced sequencing technologies. Annu Rev Genomics Hum Genet 2017; 18: 229-256.

30 Best DH, Sumner KL, Smith BP, et al. EIF2AK4 mutations in patients diagnosed with pulmonary arterial hypertension. Chest 2017; 151: 821-828.

31 Hadinnapola C, Bleda M, Haimel M, et al. Phenotypic characterization of EIF2AK4 mutation carriers in a large cohort of patients diagnosed clinically with pulmonary arterial hypertension. Circulation 2017; 136: 2022-2033.

32 Wooderchak-Donahue WL, McDonald J, O’Fallon B, et al. BMP9 mutations cause a vascular-anomaly syndrome with phenotypic overlap with hereditary hemorrhagic telangiectasia. Am J Hum Genet 2013; 93: 530-537.

33 Wang G, Fan R, Ji R, et al. Novel homozygous BMP9 nonsense mutation causes pulmonary arterial hypertension: a case report. BMC Pulm Med 2016; 16: 17 .

34 Long L, Ormiston ML, Yang X, et al. Selective enhancement of endothelial BMPR-II with BMP9 reverses pulmonary arterial hypertension. Nat Med 2015; 21: 777-785.

35 David L, Mallet C, Mazerbourg S, et al. Identification of BMP9 and BMP10 as functional activators of the orphan activin receptor-like kinase 1 (ALK1) in endothelial cells. Blood 2007; 109: 1953-1961.

36 Ricard N, Ciais D, Levet S, et al. BMP9 and BMP10 are critical for postnatal retinal vascular remodeling. Blood 2012; 119: 6162-6171.

37 Tillet E, Ouarné M, Desroches-Castan A, et al. A heterodimer formed by bone morphogenetic protein 9 (BMP9) and BMP10 provides most BMP biological activity in plasma. J Biol Chem 2018; 293: 10963-10974.

38 Chen H, Brady Ridgway J, Sai T, et al. Context-dependent signaling defines roles of BMP9 and BMP10 in embryonic and postnatal development. Proc Natl Acad Sci USA 2013; 110: 11887-11892.

39 Briggs LE, Phelps AL, Brown E, et al. Expression of the BMP receptor Alk3 in the second heart field is essential for development of the dorsal mesenchymal protrusion and atrioventricular septation. Circ Res 2013; 112: $1420-1432$.

40 Kumar ME, Bogard PE, Espinoza FH, et al. Mesenchymal cells. Defining a mesenchymal progenitor niche at single-cell resolution. Science 2014; 346: 1258810.

41 Xie T, Liang J, Liu N, et al. Transcription factor TBX4 regulates myofibroblast accumulation and lung fibrosis. J Clin Invest 2016; 126: 3063-3079.

42 Malzer E, Dominicus CS, Chambers JE, et al. The integrated stress response regulates BMP signalling through effects on translation. BMC Biol 2018; 16: 34. 